\title{
SECURITY DYNAMIC \\ OF THE STRATEGIC NUCLEAR BALANCE IN SYSTEMS THEORY AND THE CONCEPT OF CENTRES OF GRAVITY
}

\author{
Mario MARINOV, \\ Ph.D. Candidate, University of Library Studies and Information Technologies, \\ Sofia, Bulgaria. \\ E-mail: m.marinov@unibit.bg \\ Plamen BOGDANOV, Ph.D., \\ Brig.-Gen. (ret.), Associated Professor, \\ University of Library Studies and Information Technologies, Sofia, Bulgaria. \\ E-mail: p.bogdanov@unibit.bg
}

\begin{abstract}
The general concepts and understandings of the international security environment in the 21 st century have become more complex owing to a multitude of factors spanning both traditional and novel security domains. In the current era, nuclear security in particular has continued to exist in its paramount role of importance. The following paper explores the defined state of the "strategic nuclear balance" as a state extant within the systemic approach towards international security. Based upon previous research in defining the state through core concepts fundamental to systems theory and supporting concepts such as those of centres of gravity, this paper aims to further expand contemporary understandings and illustrate a point of coalescence. Thereby the given study demonstrates how based upon key postulations in the understanding of the security concept in systems theory and centres of gravity of both physical and non-physical nature within a system, a combined novel model can be extrapolated, which demonstrates the security dynamic in the "strategic nuclear balance" between major global nuclear armed state actors.
\end{abstract}

Keywords: strategic nuclear balance; centres of gravity; systems theory.

\section{Introduction}

The complex international security environment of the 21 st century offers a diverse and ever-expanding array of challenges in maintaining peace and stability, ranging from the traditional domains of warfare and the novel threats that have come to permeate them to the technologically revolutionary new domains of space and the digital realm. The theoretical application of models is vital in establishing a coherent dynamic that can explain past, present and emerging security phenomenon, as well as in anticipating their effects. Amongst the challenges to security, stability and peace, and a subject area of particular and continuing interest for all major global actors, is the nuclear security sphere, whose continued importance has not dwindled since its apogee during the latter half of the Cold War, and which now stands once again as the preeminent factor of importance in shaping the strategic landscape of tomorrow. In providing for future stability the subject matter of nuclear security, necessitates continued discussion and theoretical analysis encompassing the examination and superposition of past such efforts with contemporary ones. 
STRATEGIES XXI International Scientific Conference

The Complex and Dynamic Nature of the Security Environment

The following paper serves the primary purpose of further expanding previous research into the theoretical understanding of the "strategic nuclear balance" in systems theory and the notions therein of "security" as a fundamental state of a system, where the "strategic nuclear balance" has previously been defined as the extant moment security state derived in the international system of relations from the security interactions of the constituent sub-systemic elements of the national subsystems. These national subsystems in the international system are considered to be foremost global nuclear powers, which possess the highest degree of potential to precipitate change in the international system, and are themselves viewed as complex recursive systems possessing other complex systems within them, and which through their own interactions and structure coalesce into the operations and capabilities of the larger national subsystem. The paper further expands upon previous efforts in collaborating the security environment dynamic in the "strategic nuclear balance" through the implementation of the concept of Centres of Gravity (CoGs), as fundamental in understanding the security state on both the highest order systemic level of the international system, and the recursive national subsystem levels within it.

Thusly, the following paper has as its main object of theoretical analysis the "strategic nuclear balance", with the principal subject being the further implementation and coalescence of systems theory and the concepts of Centres of Gravity in a more precise and clearer model, showcasing key dependencies and set dynamic.

The working thesis of the paper is that based upon the key elements of zones and levels of security in the systems-based approach towards international relations, as well as the concept of centre of gravity, a model of the evolution of the "strategic nuclear balance", which this paper has dubbed the "Hourglass Model", can be established. This model showcases the dynamic between levels of security and Centres of Gravity of two national subsystems, the dynamic of their transition within the international systems and within different zones of security, creating points of balance, or points of great disbalance, where security risks and threats are at their highest values.

In its structure the paper will adhere to the following methodology:

- The paper will first examine the notion of "security" as a dynamic state based on systems theory, utilising the additional notions of levels and zones of security as key components in understanding its exact dynamic across the systemic levels of the "strategic nuclear balance";

- The paper will then reiterate the key points of CoGs within the individual systemic levels of the "strategic nuclear balance", as well as their expression;

- Finally, the paper will seek to establish a model of interactions between CoGs and levels and zones of security, demonstrating their mutual dependencies and overall dynamic.

In exploring the notions of the "strategic nuclear balance" the paper will adhere to several limitations for the purposes of narrowing down its scope. Within systems theory and the exploration of the concept of "security" as an element within the international system, the following paper will not focus on the individual examination of the concept within specific national subsystems, where perceptions and definitions of "security" inevitably vary, but will instead focus on "security" as a state or condition of the system/s based upon the key requirements for overall existence, regardless of the examined national security system. Furthermore, the examination of the "strategic nuclear balance" within this approach will primarily focus on the dynamic between the highest order systemic level of the international system and the constituent lower order systemic levels of the national subsystems. These specific interactions are considered to be of the highest value in establishing a dynamic within the "strategic nuclear balance". The national subsystems, when examined through systems theory are in their own right complex recursive systems possessing other lower order systems, 
which make up their structure and operation, offering additional avenues of exploration in further defining systemic behaviours, but are outside the scope of the present paper. Additionally, when examining the "strategic nuclear balance", which traditionally entails the exploration of the specific historical nuclear-superpowers and their specific security concerns within the international system, the following paper will instead focus on providing the theoretical basis for a model of the dynamic within the "strategic nuclear balance", where the constituent nuclear superpowers will simply be referred as Subsystem A and Subsystem B, or overall the national subsystems within the international system. Finally, for the purposes of this paper, an in reiteration of the previously stated chosen approaches towards the analysis of the "strategic nuclear balance", it is defined as "a dynamic state with moment values within a given time interval and within the framework of a complex system of interactions. This complex system of interactions is at its highest order the "international system", and is constituted by interacting recursive hierarchical complex subsystems, with the primary such subsystems and the ones most vital in forming the "strategic nuclear balance" being the national subsystems."

\section{Levels and Zones of Security in Systems Theory and the "Strategic Nuclear Balance"}

In understanding the "strategic nuclear balance" through the lens of systems theory, of paramount importance are the concepts of "security" and the "security state", as the particular condition in terms of security perceptions of a system at any specific time. However, "security" is an ambiguous concept with widely varying definitions and perceptions across specific nations, languages, cultures and historical periods, as examined within the complementary organisations-based approach towards understanding the behaviour of systems. The task of its precise understanding, definition, and implementation is a daunting challenge, and requires an individualistic approach towards its exploration in each individual national subsystem level, and thus falls outside of the scope of the current paper. Instead of attempting to provide such a definition of "security" for each systemic level, which will inevitably vary in its precise content, within systems theory and specifically the operation and dynamic of a security system, the following paper will employ the terminology established by E. Manev for the set of primary elements employed in constructing the "security" concept and the same such elements necessary for its continued function (Manev et al, 2017, 33-34):

- Security levels - moment values indicating the probability of unwanted events occurring, which have the high probability of decreasing the nominal functioning of the system.

- Security zones - intervals, within which the security levels correspond to set requirements and within which the security level can vary between given boundaries.

- Normal state - the state when a system can function under its established organisational, architectural and functional model;

- Nominal state - the state when a system functions closest to its predicted optimal state, thereby possessing the maximum possible security level.

- The security dynamic is further expanded in the specific characteristics of the three defined zones of operations for a given system and its subordinated subsystems (Manev 2016, 265):

- Normal security zone - where the system operates within its defined nominal state and parameters of operations, and where the security level can deviate in expected and manageable margins, close to the optimal state, as such in this zone the system is guaranteed the highest level of security and there are no apparent risks for its structural integrity.

- Emergency security zone - where the system, due to the emergence of factors has passed outside of the normal zone of operations, and therefore experiences heightened risks and 
STRATEGIES XXI International Scientific Conference

The Complex and Dynamic Nature of the Security Environment

threats to its integrity, thus producing a lowered security level. In this zone the systems undertake planned steps to return to the normal zone of operations.

- Catastrophic security zone - where the system's integrity and operations have been degraded to the extent where operations are no longer possible and there are insufficient resources within it to restructure itself and return to the normal zone. In this zone the system can no longer provide for the levels of security for continued existence. Past this zone the system faces either complete collapse, or complete restructuring through outside intervention and input of resources.

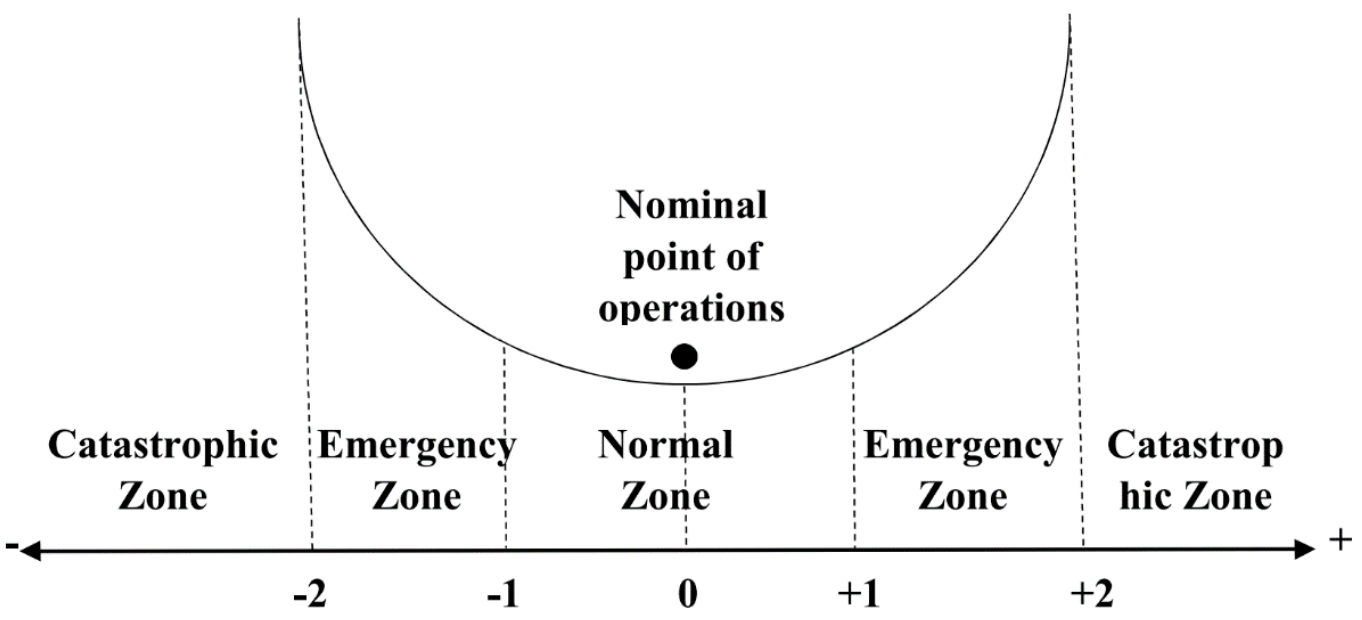

Figure no. 1: Zones and Levels of Security (Manev et.al. 2017, 35)

When the system transitions from one zone to the other it follows the dynamic of moving from one security level to the other (as numerically abbreviated in the below graphic [Fig.1]), potentially progressively moving from the highest security level, where there is a lack of direct threats or risks, towards a lower security level (the lowest being the catastrophic zone where the system is overwhelmed), or rebounding to again return to a higher security level (Manev et al 2017, 34).

In the international system and the systems-based approach towards understanding the "strategic nuclear balance", the "normal zone" and "normal state" of operations can be considered to revolve around the primary systemic objective of structural survival of any given system. In the "normal zone" and within the notion of the "strategic nuclear balance" such a normal state of ensured survival can be constructed around the concept of "deterrence" where all constituent national subsystems must find themselves in a state of equally perceived levels of deterrence and equally accepted notions of "mutually assured destruction" (Marinov 2020 (1), 770-773). When such conditions exist, both the international system and the national subsystems can be stated to have achieved a nominal state and level of security, as the threats of destabilisation and collapse at all systemic levels are at their lowest values. Such a state has historically been further defined in specific qualitative and quantitative values in nuclear arms striving towards mutually acknowledged parity, and has been the desired state for the nuclear super-powers in previous decades (Marinov 2020 (2), 66-67).

Additionally, the following additional notions must be taken into account in the specifics of the "strategic nuclear balance", when precipitating change in systemic levels, namely (Marinov 2020 (2), 66-67):

- The nominal and optimal levels of security in the highest order system - the international system, are subject to the actions and interactions between the national 
subsystems. Therefore, the actions of one subsystem can unliterary and significantly alter the overall state of the international system and its levels of security.

- Any given national subsystem can proceed to have a defined "optimal security level" that is drastically different from the moment values of the security level in the international system. When such an occurrence takes place there are immediate consequences for both the levels of security in the international system, and the corresponding other national subsystems, which will also strive to change their respective security levels to ensure a return to the "normal zone" for their respective operations, thus continuing and propagating the effects of change and transition in the zones of security in the international system, pushing the higher order system towards either further destabilization or coalescence around a new "normal state" of operations for all systems [Fig. 2]. Such a dynamic can be stated to also correspond in part to the concept of the "security dilemma" in the realist school of examining international relations, where various extrapolations of state behaviour and eventual conflict evolution have also centred on the notions of mutual fear. However, a clear distinguishing characteristic of the approach proposed here is that it offers a more holistic method towards defining an overall dynamic and specifically the necessary context for establishing rules and preconditions for conflict prevention in the nuclear era. In conjunction with the organisational approach, such an approach towards nuclear security in particular are the individualised and specific extrapolations of national subsystem behaviour based upon their specific organisational characteristics and security perceptions.

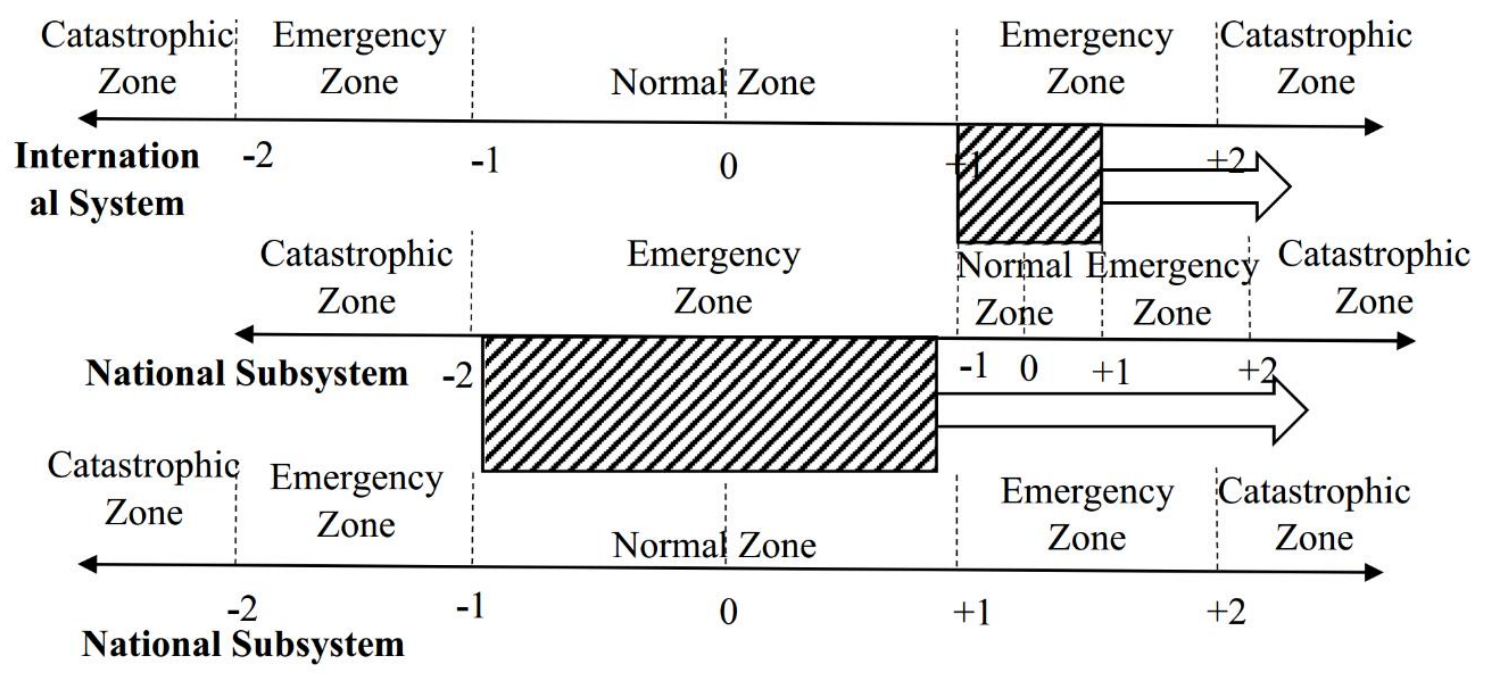

Figure no. 2: Levels and Zones of Security in Systemic relation in the "Strategic Nuclear Balance" (Marinov. 2020 (2), 66)

\section{Centres of Gravity in the Strategic Nuclear Balance}

Centres of Gravity stand as the next vital concept in constructing a coherent model for the security dynamic of the "strategic nuclear balance". CoGs in their own right have been a vital notion in projecting and understanding the balance of military power between adversaries, ever since Clausewitz first established the concept in the 19th century. The concept, its precise definition and implementation in modern military strategy, remains a topic of heated discussion to this day. CoGs have been postulated to possess both physical and non-physical characteristics, pertaining both to one's own capabilities and those of adversary, and constituting the elements, which when neutralised would either significantly reduce military capabilities and or possess the ability to achieve victory in tactical, operational and strategic terms, as per M. Vego's definition: ,, a source of “massed” strength - physical or moral - or a 
STRATEGIES XXI International Scientific Conference

The Complex and Dynamic Nature of the Security Environment

source of leverage, whose serious degradation, dislocation, neutralization, or destruction would have the most decisive impact on the enemy's or one's own ability to accomplish a given military objective; tactical, operational, and strategic (theatre-strategic and national/alliance/coalition) CoGs are differentiated; each CoG is related to the corresponding military objective to be accomplished" (Vego 2017, 43).

CoGs also have systemic characteristics, where the degradation of the systemic connections and the stability of a given system would be rendered inoperable the adversary's or one's own military capabilities, as per A. Echevarria: „, CoG is not the strength, not the source of strength and not a weakness. CoG is what holds the enemy's force together. CoG is the "focal point" that holds the system together, but only exists if there is a certain degree of connection" (Echevarria 2002, 16).

Both concurrent definitions are applicable in understanding the security dynamic of the "strategic nuclear balance" albeit at different systemic levels and in consideration of the specific characteristics of these levels.

On the national subsystem level, CoGs constitute the physical elements of strategic nuclear power. However, unlike traditional CoGs, which manifest in a conflict and whose effectiveness is intrinsically tied with effectively destroying the adversary's $C o G$, whilst protecting one's own, in the "strategic nuclear balance", the inherent vulnerability to the farreaching structure of two complex national subsystems afforded by strategic nuclear arms is the ultimate instrument in ensuring their individual survival through the concept of deterrence. The greater the disparity in capabilities between the two subsystems, the greater the associated threat level that will be perceived by the one side. Consequently, and in the interest of mitigating security concerns on the level of the international system, national subsystems have historically moved towards strictly defining their individual $C o G s$, creating an environment of mutual predictability, and placing limitations and values on their qualitative and quantitative characteristics, creating the idea of nuclear parity, as a quintessential element of strategic nuclear stability and consequently the "strategic nuclear balance". Such processes and the very concept of negotiating power distribution and parity through a treaty system are not confined to the history of the "strategic nuclear balance" and modern international relations, but are the historical result and principal method of operation and interaction of social organisations in general, which the national subsystems examined can be defined to be. The phenomenon has been prevalent in previous historical periods - the Washington and London Naval Treaties sought to achieve such results in the international system in qualitative and quantitative terms for capital ships, which were considered the mechanism for strategic power projection of that era; the preceding era of the "Bismarck system" sought to produce contrasting alliances of equal power level in balancing European powers and their global ambitions; even in the historical record of treaties between the Roman and Persian Empires, the idea of territory distribution in the border-regions of the two empires is centred around parity in spheres of influence. In the nuclear era the major global nuclear super-powers utilise the instrument of the bilateral treaty obligations and specific limitations to establish a working "nuclear treaty regime" again centred on the idea of parity. This "regime" or framework of treaties, as well as the associated levels of mutual trust that form in their continued existence and application, establish the basis for the non-physical $C o G$ in the international systemic level that comes to dominate the “strategic nuclear balance" and govern its dynamic (Marinov (3) 2020, 309-310).

When in such a framework of relations, one subsystem moves to change the exact characteristics of its physical CoGs owing to a variety of factors, it establishes the preconditions to immediately degrade this non-physical $C o G$ and precipitate equal reaction from the other subsystem. Consequently, the disparity in capabilities drastically increases past this point, and 
the proportionally increases the respective security concerns and consequences for the "strategic nuclear balance".

\section{The "Hourglass" Model and the Security Dynamic in the Strategic Nuclear Balance}

Having first explored the concept of "security" through the lens of systems theory and specifically the application of levels and zones of security in extrapolating a security dynamic in the "strategic nuclear balance", as well as having examined the concept of "Centres of Gravity" and its application across systemic levels, the next logical step is to form the basis of a model that combines the efforts thus far in establishing a coherent picture of the security dynamic of the "strategic nuclear balance" across time, and which will serve as a baseline model for further studies, as well as potential additions and revisions.

The proposed model is demonstrated in the graphic below, which has been named the "Hourglass Model" [Fig. 3] and will be explained further in this section of the paper:

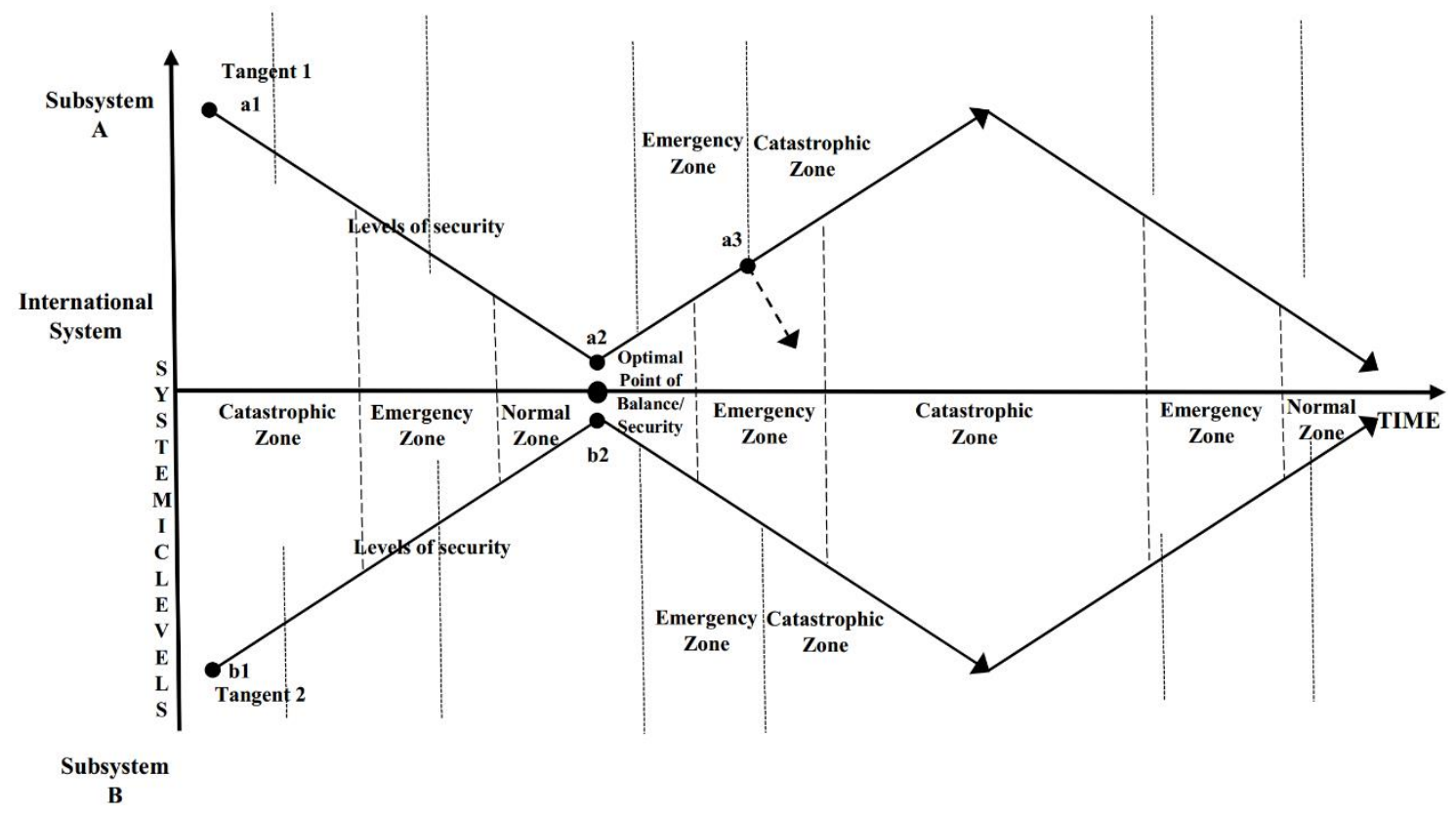

Figure no. 3: "Hourglass Model" of Zones and Levels of Security, and Centres of Gravity Interaction in Forming the "Strategic Nuclear Balance"

At its very essence the presented "Hourglass Model" is spread across two axes "Time" and "Systemic levels", where the horizontal axis demonstrates the flow of time, and vertical axis encompasses the systemic levels, examined as key to the "strategic nuclear balance", these being namely the highest level of the "International System" and the subsystem levels, or national subsystems, of the global major nuclear powers listed as "Subsystem $\boldsymbol{A}$ " and "Subsystem B". On the graphic of the model, Subsystem A is depicted above Tangent 1, Subsystem B depicted below Tangent 2, and the International System is depicted between Tangent 1 and 2, or "inside" the hourglass structure.

Across these levels of the system and across time a certain dynamic develops, which encompasses several key values of the variables spread across the graphic, and are namely: 
STRATEGIES XXI International Scientific Conference

The Complex and Dynamic Nature of the Security Environment

- Tangents 1 and 2, which form the proverbial "walls" of the hourglass and encompass both the relative levels of balance between the CoGs of the two Subsystems, as well as marking the dynamic in levels of security and the exact span of security zones across systemic levels.

- Points a1, a2, b1, b2, c1, which mark specific points in the balance between $C o G s$, as well as specific points in the levels of security shared across all systemic components.

At Points $\boldsymbol{a l}$ and $\boldsymbol{b} \mathbf{1}$, the relative disparity in capabilities between the CoGs of both Subsystems (representing, as previously stated for the national subsystems, their physical strategic nuclear power) is at its highest levels. It is also at these points that the levels of security are at their lowest values for both the subsystems, as well as for the higher order international system, and all exist in the "catastrophic zone", where the risk of a conflict breaking out is at its highest.

Subsequently and in order to increase relative levels of security in the international system and to ensure their own survival, both subsystems move to bridge the gap, aligning their security perceptions articulated in equally expressed zones of security, and advancing along both Tangents $\mathbf{1}$ and $\mathbf{2}$, towards points $\boldsymbol{a} \mathbf{2}$ and $\boldsymbol{b} \mathbf{2}$, respectively. In this dynamic the relative disbalances in CoGs are gradually eliminated, which in the "strategic nuclear balance" is seen as the establishment of a clear and mutually acknowledged state of necessary "deterrence". In historical terms, this has implied the gradual reduction of nuclear arsenals to more manageable and equal levels, elimination of weapons classes that can be detrimental to the security perceptions of both sides, the establishment of clear channels of communications in emergency scenarios, and most importantly the extension of all such efforts through the established toolset of the international system, materialising in a framework of regulatory mutual treaties, the so called "nuclear treaty regime".

This dynamic has its apogee at points $\boldsymbol{a} \mathbf{2}$ and $\boldsymbol{b} \mathbf{2}$, where the strategic nuclear power (both offensive and defensive) of both subsystems is near equal. It is also at this point that both the international system and its subsystems in their levels of security have reached a mutual optimal point of security perceptions and where the definition of an existent and true to the word "strategic nuclear balance" can exist. Within this "normal zone" of operations for the international system, the primary goals of assuring peace and stability, and therefore a near lack of security risks and threats, are achieved and maintained. The treaty regime at this point in the security dynamic, stands as the non-physical $C o G$ of the international system and thus its degradation will move the overall system again towards lower levels of security and greater disbalance.

Whilst a continued state of affairs, such as the one existing within the "normal zone" of operations for all systemic elements is preferred and desirable, it cannot be maintained in perpetuity. The even larger international security system and the organisational characteristics of national subsystems as social organisations and complex systems in their own right are extremely dynamic, and a change in overall security policy or an increased perceived threat in one subsystem from the other, results in that subsystem's security perceptions and corresponding levels of security changing values. Such a perceived threat could also come from other subsystems in the international system, which over time could have advanced the capabilities to constitute a new factor in the "strategic nuclear balance" and its dynamic. Consequently, a Subsystem in such conditions would seek to expand its direct nuclear capabilities, changing the characteristics of its $C o G$, and diverging from the optimal point of balance with the other principal Subsystem, thus precipitating equal requirements for change within it. However, such a change in the physical CoGs of both Subsystems would entail a degradation of the non-physical $C o G$ of the international system, the "nuclear treaty regime", which seeks to govern Subsystem CoGs, pushing the international system towards the emergency zone, and finally into the catastrophic zone. 
In this latter half of the dynamic, and particularly in the "emergency zone", a Subsystem could undertake steps, as in Point a3 to return to a point of balance, thus serving as the basis for the potential emergence of a new "normal zone". However, in an environment permeated by a lack of mutual efforts, or in the case of the emergence of a new significant subsystem (a new major strategic nuclear power), as well as a subsystem unwilling to effectively engage with its peers, the outcome is a clear transition to the "catastrophic zone" and the collapse and subsequent reconstruction of the international system through significant global geopolitical changes.

\section{Conclusion}

This final process in the demonstrated model of the security dynamic of the "strategic nuclear balance" has in the past constituted significant historical outcomes in the international security system - the established system of the international power balance (the "Alliance system") prior to 1914 and its inability to overcome a moment of crisis resulted in World War I; the subsequent system of the League of Nations, and its constituent Washington and London Naval Treaty regimes could not in the end overcome significant systemic challenges to preserving international peace and preventing an arms race, resulting in their collapse. The consequences for the "strategic nuclear balance" from a general transition to the catastrophic zone are far more severe. Uniquely and conversely to the above episodes within the international system, the gravity of nuclear weapons and the outcomes of their perspective employment have historically transitioned the system through variable points of emergency in the past seven decades. Within the proposed model, of interest are the effects of introducing a third significant national subsystem, making the observed model from a two-dimensional one to a three-dimensional one. The effects of continued technological progress, the further militarisation of space and the development of both novel offensive and defensive strategic weapons systems, are also vital in further extrapolating the future dynamic of the "strategic nuclear balance" and the direct effects to subsystem CoGs and security perceptions. The behaviour of the specific national subsystems in the international system also depends of numerous specific and unique organisational characteristics pertaining to the very essence of the security concept. All of the above; however, fall outside of the scope of this paper, and would serve as the basis for the future expansion and refinement of the proposed "Hourglass" model, which has thus far combined key approaches in understanding the security dynamic in the international system through systems theory, the specifics of the "strategic nuclear balance" within it, as well as the associated Centres of Gravity that develop and evolve around the main systemic elements.

\section{BIBLIOGRAPHY:}

***, Глобална, регионална и национална сигурност [author's translation: Global, Regional and National Security]

***, Определение за сигурост - организационнокурурен подход [author's translation: Definition of Security - an Organizational-Cultural Approach]

***, Системен подход за анализирането на съвременния стратегически ядрен баланс като елемент на международната система [author's translation: Systemic Approach for Analyzing the Contemporary Strategic Nuclear Balance as an Element of the International System]

ECHEVARRIA, Antulio. 2002. Clausewitz's Center of Gravity: Changing Our Warfighting Doctrine-Again!: U.S. Army War College. 
MANEV, Evgeni, et al. 2016. “Определение за сигурост - организационнокурурен подход", Burgas: Journal of Legal Studies, Vol. XXIII.

MANEV, Evgeni. 2012. Глобална, регионална и национална сигурност, Sofia: Softtrade.

MARINOV, Mario. 2020. "Defining Centres of Gravity within the Strategic Nuclear Balance Between the United States of America and the Russian Federation". Bucharest: Carol I Nation Defence University.

MARINOV, Mario. 2020. "Organisational and Systems-based Approach for Defining Levels and Zones of Security within the Strategic Nuclear Balance between the United States of America and the Russian Federation". Sofia: Security - Education, Science, Industry, Georgi Rakovski Military Academy.

MARINOV, Mario. 2020. “Системен подход за анализирането на съвременния стратегически ядрен баланс като елемент на международната система”. Sofia: Journal of Knowledge Society and 21st Century Humanism, University of Library Studies and Information Technologies, Sofia.

VEGO, Milan, Joint operational warfare: theory and practice, in Ion Chiocea et al. 2017. "Definitions of Center of Gravity - Evolution and Interpretations, Technologies". Bucharest: Military Applications, Simulation and Resources. "Carol I" National Defence University. 